\title{
Der Markgraf Rüdiger von Bechelaren im Nibelungenlied und Rodingeir von Bakkalar in der Thidrekssaga. Figurenporträt, Vergleich und Höfisierung
}

\section{The Nibelungenlieds Margrave Rüdiger von Bechelaren and the Thidrekssagas Rodingeir von Bakkalar. Portrait, Comparison and Adaptation Courtoise}

\author{
Martha Christine Süß' (D)
}

${ }^{1}$ Master of Arts, Ruprecht-Karls-Universität, Germanistisches Seminar, Heidelberg Germany

ORCID: M.C.S. 0000-0002-2421-6565

\section{Corresponding author:}

Martha Christine Süß,

Master of Arts, Ruprecht-Karls-Universität, Germanistisches Seminar, Heidelberg Germany, Post adress: Kaiserstraße 11 66849 Landstuh

E-mail: christine.suess@yahoo.de

Submitted: 15.02 .202

Accepted: 11.04.2021

Citation: Süß, M. C. (2021). The Nibelungenlieds Margrave Rüdiger von Bechelaren and the Thidrekssagas Rodingeir von Bakkalar. Portrait, Comparison and adaptation courtoise. Alman Dili ve Edebiyatı Dergisi - Studien zur deutschen Sprache und Literatur, 45, 29-49. https://doi.org/10.26650/sds|2021-871818

\section{ABSTRACT (DEUTSCH)}

Das Nibelungenlied und die Thidrekssaga stellen zwei Aktualisierungen desselben Stoffes dar, und zeigen doch eine Reihe an Unterschieden. Der vorliegende Artikel beleuchtet die Figur, die das Nibelungenlied Markgraf Rüdiger von Bechelaren, die Thidrekssaga Rodingeir von Bakkalar nennt. Er erstellt ein Figurenportrait und eine Forschungsübersicht, während die Funktionen, die die Figur in beiden Texten für deren Strukturierung übernimmt, extrapoliert werden. Hierbei wird ersichtlich, dass das Nibelungenlied anhand der Figur Rüdigers mittels des Erzählerverhaltens auf der einen, und durch den Einsatz von bekannten Elementen hochmittelalterlichhöfischer Literatur, beispielsweise durch das Brautwerbungsschema, des locus amoenus, durch Konzepte des Tugendrittertums, und die Kontrastierung von Werten christlicher und weltlicher Lebensführung, eine Höfisierung (adaptation courtoise) des gesamten Textes konstruiert. Rüdigers Funktion als Brautwerber Etzels führt im Nibelungenlied gar dazu, dass das Werk als helferzentrierte Brautwerbungsdichtung gelesen werden kann - trotz des Umstands, dass der Markgraf keine Hauptfigur darstellt, kommt ihm eine signifikante Bedeutung für den Gesamttext zu. Dieser höfische Überbau bleibt in der Thidrekssaga aus: Im Nibelungenlied begegnet im Markgrafen Rüdiger ein tugendhafter Ritter in nuce, während er in der Thidrekssaga rein funktionalen Charakter bewahrt und an den Stellen auftritt, die seiner für den Fortgang des Plots bedürfen. Das vorbildliche Rittertum Rüdigers führt schließlich zu dem bekannten Vasallitätskonflikt, in dem eine Hierarchisierung der vasallischen Bindungstypen nur scheinbar Abhilfe schaffen kann. Im Zuge dieses Konflikts werden an der Figur des Markgrafen außerliterarische Konflikt verhandelt. Der Artikel demonstriert, dass die Figur im Nibelungenlied eine zentrale, bedeutungsund strukturbestimmende Bedeutung innehat, während sie in der Thidrekssaga marginal und funktionalisierend genutzt wird.

Schlüsselwörter: Nibelungenlied, Thidrekssaga, Brautwerbungsschema, Höfisierung, christliche und weltliche Lebensführung 


\begin{abstract}
ENGLISH)
The Nibelungenlied and the Thidrekssaga are two actualizations of the same plot, yet they show a number of differences. By outlining the portrait of the protagonist the Nibelungenlied calls Margrave Rüdiger von Bechelaren and the Thidrekssaga calls Rodingeir von Bakkalar, while reviewing pre-existing literature on the subject, the article at hand demonstrates the figure's vast differences. In doing so, the article demonstrates that in the Nibelungenlied, an adaptation courtoise (Höfisierung) is achieved through various characteristics of the Margrave, such as the use of a specific narration mode, the employment of the Topos locus amoenus, and the contrasting reflection of Christian and worldly lifestyles and their values. The Nibelungenlied can even be classified as a helferzentrierte Brautwerbungsdichtung (a piece of helper-focused courtship poetry) because of the Brautwerbungsschema (courting scheme) used by Rüdiger to find a wife for his feudal lord Etzel. The Margrave does not pose as a main protagonist, overall, he is still of high importance to the Nibelungenlied. These courtoise elements are absent in the Thidrekssaga. While Rüdiger appears as a model-knight in the Nibelungenlied, his counterpart, Rodingeir, in the Thidrekssaga plays a strictly functional role in advancing the plot. In the Nibelungenlied, the protagonists status as a model-knight culminates in the well-known vasallity conflict, in which a hierarchization of types of vassal obligations only seemingly settles the conflict. Moreover, through this dilemma, the non-literary conflict of Christian and worldly lifestyles is negotiated. The article at hand demonstrates that Rüdiger von Bechelaren's character is integral to the meaning and structure of the Nibelungenlied and determines the genre, while Rodingeir von Bakkalar is only employed marginally and functionally.
\end{abstract}

Keywords: Adaptation courtoise, medieval literature, germanic heroic epic poetry, Dietrichepik, Tugendrittertum

\title{
EXTENDED ABSTRACT
}

The Nibelungenlied and the Thidrekssaga are two actualizations of the same plot, yet they show a number of differences, for example the depiction of one of their protagonists. By outlining the portrait of the protagonist the Nibelungenlied calls Margrave Rüdiger von Bechelaren and the Thidrekssaga calls Rodingeir von Bakkalar, as well as reviewing preexisting literature on the subject and identifying possible historic models or prefigurations, the article at hand demonstrates the figure's vast differences. Additionally, it shows that in the Nibelungenlied, the figure serves as a genre defining and plot-driving force and helps to adapt the older heroic matters and plots circulating around the figure of Dietrich von Bern to the German High Middle Ages courtly literary style, while Margrave Rodingeir takes a minor role in the Thidrekssaga. The article demonstrates that in the Nibelungenlied, an adaptation courtoise (Höfisierung) is achieved through various characteristics of the Margrave, such as the use of a specific narration mode, the employment of the Topos locus amoenus, and the contrasting reflection of Christian and worldly lifestyles and their virtues. The Nibelungenlied can even be classified as a helferzentrierte Brautwerbungsdichtung (a piece of helper-focused courtship poetry) because of the Brautwerbungsschema (courting scheme) used by Rüdiger to find a wife for his feudal lord Etzel. This scheme consists of specific elements that can be employed as a group or individually and describe the process of getting married or helping someone else get married: Deciding a marriage shall take place, courting the lady in 
question, getting married in her native country and the following repatriation of the bride to the grooms dominion after the marriage can all be part of the scheme. Even though the Margrave does not pose as a main protagonist, overall, he still is of high importance to the Nibelungenlied, as he acts as a genre deciding factor and drives the Höfisierung (adaptation courtoise). These courtoise elements are absent in the Thidrekssaga. While Rüdiger appears as a model-knight abiding by all the knightly virtues such as triuwe, mâze and staete in the Nibelungenlied, his counterpart, Rodingeir, in the Thidrekssaga, plays a strictly functional role in advancing the plot. The Margraves knightly virtues show in the figures behaviour, including among others the receptions and treatment his guests receive at the Margraves court function. These act as a sign of the differing depiction of the figure in both works. It is at his dominion that the marriage of Rüdigers daugther and the Burgundian Giselherr is agreed upon in a scene full of elements belonging to the courtly literary style of the European High Middle Ages such as the strict following of courtly ceremony while receiving, entertaining and seeing guests off, as well as great hospitality and hospitality gifts. Furthermore, this courtly reception does not just emphasize Rüdigers model behaviour and knightliness, but is also an example of the Höfisierung or adaptation courtoise of the Nibelungenlied as a whole that is consummated by the Margrave. Moreover, in the Nibelungenlied, the obligation arising from the newly forged family ties that arise from the wedding of Rüdigers daugther and Giselherr together with the Margraves status as a model-knight culminate in the well-known vasallity conflict, in which the figure is trapped in a battle between his feudal lord and his in-laws. A hierarchization of types of vasall and moral obligations only seemingly settles the conflict. The Margrave now not only belongs to the sphere of Etzel, who is his feudal lord, but also to the Burgundian sphere, who he is related through by marriage. The protagonists legal obligations towards his lord and the moral and virtuous implications of his ties forged by marriage cannot be reconciled. Through Rüdigers dilemma, the non-literary conflict of Christian and worldly lifestyles is negotiated, as the article at hand shows. The heroic Nibelungenlied epic asks how concepts of worldly order such as the feudal system and Christian beliefs can be combined into a knightly lifestyle and therefore fits in with the negotiation of traditional heroic virtues and behaviours and the relatively new concepts of Christianity. The article at hand demonstrates that Rüdiger von Bechelarens character is integral to the meaning and structure of the Nibelungenlied and determines the genre, while Rodingeir von Bakkalar is only employed marginally and functionally. 


\section{Schemata und Erzählerverhalten - das Thema dieses Artikels}

Dieser Artikel befasst sich mit der Figur, die das Nibelungenlied (NI) Rüdiger und die Thidrekssaga (Ths) Rodingeir nennt. Er fragt nach einem möglichen historischen Vorbild, um anschließend Darstellung und Funktion der Figur in beiden Texten zu untersuchen. Auffallend ist zunächst die unterschiedliche Gestaltung beider Texte. Zentral wirkt hier der unterschiedliche Grad der nach mittelhochdeutsch-höfischen Literaturstandards definierten Höfisierung beider Aktualisierungen des Stoffes. Dem Konzept der Höfisierung ${ }^{1}$ konstitutiv ist der Versuch der Anpassung von Stoff und Motiv an den „Vorstellungshorizont und [die] Realitätserfahrung [der] Zeitgenossen" (Schulze, 1997, S. 142). Hierzu werden im Falle des $N I$ "kulturelle Errungenschaften der Adelsgesellschaft aus der zweiten Hälfte des 12. Jahrhunderts und spezielle Vorgaben der neuen höfischen Literatur" (Schulze, 1997, S. 142) genutzt. Das Ergebnis dieses Verfahrens ist eine "adaptation courtoise" (Schulze, 1997, S. 142). Der geringeren Funktionalität des Markgrafen in der Ths steht eine für Handlungsgang und Wirkung auf den Rezipienten unverzichtbare im NI gegenüber: Obwohl der Markgraf hier keine Hauptfigur ist, kommt ihm eine zentrale Funktion für den Gesamttext zu. Rüdiger trägt zentral zu der bereits angesprochenen Höfisierung bei: sie wird einerseits durch das Verhalten des Erzählers und die daraus folgende Figurengestaltung ermöglicht. Hieraus ergibt sich dann höfisierungskonstitutiv die Möglichkeit, während des Figurenkonflikts die Frage zu reflektieren, wie weltlich-diesseitige Ordnungsvorstellungen mit christlich-jenseitigen der höfischen Gesellschaft des Hochmittelalters vereinbar sein können. Andererseits wird der Text durch den Einsatz literarischer Elemente und Schemata der mittelhochdeutsch-höfischen Literatur in Zusammenhang mit der Figur des Markgrafen strukturiert. Es finden sich also zwei Ebenen der Höfisierung: Erstens das Erzählerverhalten und die Ausführung seiner Möglichkeiten an der Figur und zweitens die Implementierung dem Rezipienten allgemein bekannter Elemente höfischer Literatur, denen werkkonstitutiver Charakter zukommt - beide Ebenen finden ihren Fixpunkt in der Figur des Markgrafen, ermöglichen den finalen Konflikt der Figur, und leiten auf ihn hin.

\section{Die Überlieferung der behandelten Texte}

Wie bereits angedeutet, befasst sich der Artikel mit zwei Vertretern der Nibelungenund Sigfried-Dichtung, der Ths und dem NI, die stofflichen Überschneidungen, gleichzeitig Unterschiede aufweisen, denen sich der Artikel später widmet.

1 Der Begriff,Höfisierung' ist in diesem Artikel an die Definition in Schulze, Ursula (1997). Das Nibelungenlied. Stuttgart: Reclam, S.142, und Breulmann, Julia (2009). Erzählstruktur und Hofkultur. Weibliches Agieren in den europäischen Iweinstoff-Bearbeitungen des 12. bis 14. Jahrhunderts. Münster: Waxmann, S.97, angelehnt. 
Ist die in der norwegischen Hansestadt Bergen Mitte des 13. Jahrhunderts (KramarzBein, 2002, S. 1) aufgezeichnete Ths „als bloße Übersetzung eines niederdeutschen Originals" (Kramarz-Bein, 2002, S. 2), oder "als eine altnorwegische Schöpfung" (KramarzBein, 2002, S. 2) zu betrachten? Sie ist in zwei vollständigen Pergamenthandschriften (Mb und Sv), zwei Abschriften (Is Hs A/B), sowie mehreren Fragmenten überliefert (RitterSchaumburg, 1981, S. 32). Einigkeit besteht darüber, dass die Quellen der Saga niederdeutsche waren, seltener wird auf romanische Herkunft verwiesen (Kramarz-Bein, 2002, S. 1). Es finden sich die meisten der Stoff- und Sagenkreise, die auch im NI vertreten sind, wobei der Fokus auf der Gestalt des Dietrichs von Bern liegt. In den Verlauf der Saga werden „nahezu alle bekannten Helden und Heldinnen der germanischen Heldensage [...] einbezogen“ (Kramarz-Bein, 2002, S. 1). Das vermutlich um 1200 (Schulze \& Grosse, 2011, S. 929) in Passau (Wapnewski, 1960, S. 71) niedergeschriebene NI bildet im deutschen Sprachraum die älteste Dichtung des Nibelungenstoffkreises (Schulze \& Grosse, 2011, S. 931). Es ist in „36 mehr oder minder vollständigen Handschriften bzw. Fragmente[n]“ (Müller, 2015, S. 48) überliefert; vollständig in drei Handschriften - A, B und C (Schulze \& Grosse, 2011, S. 938). Im NI vereinen sich Sagen- und Stoffkreise verschiedenen Inhalts und Alters. Es wirken die Brünhild-, und Burgundensage, sowie Teile der Lieder-Edda (Wapnewski, 1960, S. 71; Müller, 2015, S. 32, 35). Die Frage nach dem Verhältnis beider Texte beschäftigt die Forschung seit der Lachmann'schen Liedertheorie. Panzer schließt, "[d]aß die Thidrekssaga das Nibelungenlied gekannt und benutzt hat" (Panzer, 1955, S. 276). Heusler sieht in der Ths eine Vorstufe des NI (Wapnewski, 1960, S. 71). Ähnlich wie bei der Identifikation eines Figurenvorbilds wird der Forschung „eine endgültige Lösung der komplizierten [...] stoffgenetischen Fragen [...] wohl nie vergönnt sein" (Panzer, 1955, S. 71).

\section{Zu Versuchen der Rekonstruktion eines historischen Vorbilds}

Um den historischen Kern der Figur Rüdiger zu identifizieren, haben sich viele Forscher bemüht, denn: „Eine der faszinierendsten Beschäftigungen in historischen Wissenschaften ist der Versuch, Verlorenes zu rekonstruieren" (Reichert, 1992, S. 1), und es besteht weitgehend Einigkeit darin, dass Heldensagen - zu denen das Nibelungenlied gezählt werden darf - „einen historischen Bezug“ (Heinzle, 2014, S. 11) haben. Der Figurenkern des Markgrafen jedoch verweigert „sich gleichfalls einer historischen Festlegung" (Ehrismann, 2002, S. 31). Die Figur wurde als Ableitung einer historischen Person gefasst: Von einem Grafen Rogerius berichten die Quirinalia des Metellus von Tegernsee aus der Zeit um 1160 (Metellus, 1965, S. 246). Eine weitere Nennung findet sich 
bei Herger Spervogel (Lachmann, Haupt, Vogt \& Kraus, 1970, S. 25,34-26,5). Morgan identifiziert Rüdiger mit dem spanischen Nationalhelden Rodrigo Diaz, "genannt ,el Cid campeador"' (Morgan, 1912, S. 332), Pidal schließt sich der These vom „Ebenbild Rodrigos“ (Pidal, 1937, S. 266) an. Einen ähnlichen Versuch unternimmt Kunstmann (1983, S. 233). Ebenso wurde die Figur als Produkt literarischer Arbeit gelesen. Möglich ist auch, dass sich Elemente verschiedener Herkunft und Zeitpunkte bis zur Verschriftung zusammengefunden hatten, denn:

Bestimmte Klischees alter Erzählungen werden immer wieder neu aktualisiert oder sind auf momentane Situationen jederzeit abrufbar. [...] [Der Held wird] [...] ein Konglomerat aus Volksvorstellungen, eine typische Gestalt, ein Proto-, ein Archetyp, ein Vorbild. Es dreht sich beim Volkshelden-Phänomen nicht um die reale Existenz, sondern um das Image des Idols, um den Typus, der dann - einmal etabliert - sich immer wieder neu variieren läßt. (Röhrich, 1988, S. 91-92)

So wäre die Figur ein de facto unentwirrbares Produkt verschiedenster Faktoren, vor allem: der changierenden mündlichen Überlieferung und des „kollektive[n] Gedächtnis[ses] einer schriftlosen Kriegeradelsgesellschaft" (Lienert, 2015, S. 12).

\section{Fokalisierung und Aspekte der höfisch-literarischen Gesellschaft - Bemerkungen zum theoretischen Rahmen}

Um die Figur des Markgrafen in beiden Texten beschreiben zu können, bedarf es eines Rahmens theoretischer Konzepte, der auf die Figur, ihre Funktionen und das Gesamtwerk zugeschnitten ist. Dieser Rahmen beinhaltet Schlaglichter auf narrationstheoretische Erkenntnisse und solche zur inner- und außerliterarischen Lebenspraxis der höfischen Gesellschaft, und soll bei der folgenden Textanalyse als Richtschnur gelten.

In seinem Versuch, die Methodologie des Erzähldiskurses zu fassen, postuliert Genette vier verschiedene Fokalisierungen; für die Analyse im Rahmen dieses Artikels sind zwei davon bedeutsam: die externe Fokalisierung, sowie die Nullfokalisierung. Erstere wird von Genette als der Fokalisierungstypus bezeichnet, in dem „der Held vor unseren Augen handelt, ohne daß uns je Einblick in seine Gefühle oder Gedanken gewährt würde" (Genette, 1994, S. 135), letzterer stellt die geringstmögliche "Selektion der Information gegenüber dem, was die Tradition Allwissenheit nannte" (Genette, 1994, S. 242), dar: Der Erzähler schildert die extern sichtbare Handlung und bietet oft parallel eine interne 
Perspektive. Diese gewonnenen Einblicke bilden die Basis für die Darstellung der Figurenpsychologie. Zu scheiden von der Psychologisierung der Figur durch einen Rezipienten, setzt sich diese aus „psychologischen Zuständen“ (Haferland, 2013, S. 112) zusammen, das heißt „sprachlich und kulturell kodierten Zuständen, die von Dichtern tatsächlich genannt und dargestellt werden" (Haferland, 2013, S. 112) und die einer jeden Figur konstitutiv sind (Haferland, 2013, S. 91). Nimmt man mit Haferland eine "Nullstufe der Zuteilung von Psychologie durch einen [...] Verfasser" (2013, S. 92) an eine Figur und "eine Vollstufe" (2013, S. 92) derselben an, so liegen auf dem Kontinuum zwischen beiden „anzusetzende Zwischenstufen“ (2013, S. 92). Sicher ist es richtig, anzunehmen, dass schemageleitete Literatur von mit weniger psychologischen Zuständen ausgestatteten Figuren bevölkert wird als ein moderner Roman (2013, S. 112-113); so wird die Textanalyse zeigen, dass der nullfokalisierende Erzähler des schemaorganisierten Nibelungenlieds die Figur soweit mit psychologischen Zuständen ausstattet, dass von einer Zwischenstufe ausgegangen werden muss, wobei die Darstellung der Figurenpsychologie und das Erzählerverhalten zusammenwirken.

Die „ritterlich-höfische Gesellschaft“ (Dürrenmatt, 1945, S. 19) setzt sich mit einer repräsentativ-symbolischen Handlungspraxis „ihre eigenen äußern Lebensformen“ (Dürrenmatt, 1945, S. 19), die inner- oder außerliterarisch im Konglomerat ,höfische Kultur’ zusammenfließen. Diese „äußern Lebensformen“ (Dürrenmatt, 1945, S. 19) sind insoweit äußern, als sie von dort aus betrachtbar sind. Dabei drücken sie die höfischen Tugenden aus, allen voran zuht und mâze (Dürrenmatt, 1945, S. 19). Ziel des Verhaltens nach den vorgegebenen ,äußern Formen' ist einerseits die Selbstdarstellung und inszenierung als höfisch, andererseits die Ehrerweisung "durch bestimmte Gesten, durch bestimmte Redewendungen" (Dürrenmatt, 1945, S. 19). Als bedeutende äußere Formen kommen im NI Brautwerbungssequenzen, Botenempfänge und die Darstellung ritterlicher Tugenden vor.

Die Handlungsfixpunkte des Brautwerbungsschemas fasst Schmid-Cadalbert folgendermaßen:

1) Ratsszene 2) Botenbestimmung und -fahrt 3) Hilfeverpflichtung der Dienstleute [...] 5) Gang des Werbers [...] zur Residenz des Brautvaters [...] 6) Kemenatenszene (von Werber, Bote und/oder außergewöhnlichem Helfer realisiert) [...] 9) Heimführung der Braut (Schmid-Cadalbert, 1985, S. 88) 
Das Schema des Botenempfangs besteht nach Dürrenmatt aus folgenden Elementen:

[D]ie Boten rüsten sich aus, um für ihren Herrn Ehre einzulegen, sie kommen an, werden vom Gesinde empfangen, das ihnen Herberge anweist, der Fürst erkundigt sich nach ihnen, sie treten vor die Hofgesellschaft, die sie mit bestimmten Gesten empfängt, sie bitten um Erlaubnis, ihre Botschaft ausrichten zu dürfen, man gewährt es ihnen, man ist um ihre Verpflegung besorgt, solange sie auf Antwort zu warten haben. Dieses Schema wird in jedem Einzelfall abgewandelt. (Dürrenmatt, 1945, S. 26)

Beachtenswert ist hier und für die spätere Analyse, dass Boten zu den Fürsten, denen sie die Botschaft überbringen, in der Regel eine untergeordnete Stellung einnehmen, also „ein bestimmtes Distanzverhältnis“ (Dürrenmatt, 1945, S. 26) herrscht. Obwohl keine "Systematik der höfischen Morallehre" (Bumke, 2005, S. 416) überliefert ist, lassen sich zentrale Tugenden herausarbeiten, wobei diesen „ein Fundus von christlichen Geboten“ (Bumke, 2005, S. 417) als Grundlage diente: Die Tugenden sind in ihrem Kern christliche Verhaltensregeln. Nun können als wiederholt „in poetischer Form vorgetragen[e]" (Bumke, 2005, S. 416) Tugenden diemüete, schame, kiusche, güete, triuwe, mâze und staete gelten (Bumke, 2005, S. 418-419). Die in christlichen Geboten gegebene Grundlage der ritterlichen Tugenden unterlag einem semantischen Wechsel: Bedeutete beispielsweise triuwe im ursprünglichen Sinne „die Liebe zu Gott und die Liebe Gottes zu den Menschen“ (Bumke, 2005, S. 418), so konnte ihre Reichweite innerhalb der höfischen Dichtung verringert werden: „Für den Ritter bestand die triuwe im Einhalten sittlicher Verpflichtungen" (Bumke, 2005, S. 418), als Rechtsbegriff im Einhalten der Vertragstreue des Vasallen gegenüber seinem Herrn, ebenso in der Verpflichtung, moralisch und barmherzig zu handeln (Bumke, 2005, S. 418). Über die christliche Grundbedeutung legt sich so eine weltlich-sittliche. „Daß die [...] [äußeren Formen] nicht als Selbstzweck aus der Freude an den höfischen Lebensformen gestaltet sind, ergibt sich aus ihrer Funktion für den weiteren Verlauf der Handlung" (Schulze, 1997, S. 167): Die Darstellung dieser Formen modelliert die Textstruktur mit dem Ziel der Höfisierung im NI und schafft den Boden für den späteren Konflikt. „Die Auseinandersetzung der heimischen Welt mit den neuen Gedanken [...] des Christentums [...] ist der entscheidende Vorgang der mittelalterlichen Geistesgeschichte" (Maurer, 1961, S. 6). Neben der universitären Beschäftigung mit diesem Gedankenkomplex in der Scholastik findet sich eine Thematisierung in den "germanisch-deutschen literarischen Äußerungen des Mittelalters" (Maurer, 1961, S. 6), gar eine „Verchristlichung des literarischen Lebens" (Maurer, 1961, S. 6). Während in „den heldenliedern [sic!] der Edda und in den Großerzählungen der Saga 
[...] die vorchristliche Welt" (Maurer, 1961, S. 6) dargestellt wird, findet sich seit der Karolingerzeit ein Gestalten der "heldische[n] Schicksale aus alter Überlieferung und mit christlicher Sicht" (Maurer, 1961, S. 7). Seit der Ottonenzeit (Maurer, 1961, S. 7) entwickelt sich die literarische Behandlung der Frage "was ist der Sinn unseres Daseins in der Welt und wie geben wir ihm seinen Sinn?" (Maurer, 1961, S. 8), die weltlich-höfischen Werte werden mit den jenseitig-christlichen korreliert. Dieses Korrelieren konnte bereits bei der Ausformung der Bedeutungsebenen der höfischen Tugenden beobachtet werden. Die Dichter des 13. Jahrhunderts schließlich „bejahen diese ritterliche Welt, und sie stellen sich als Christen die Frage, wie man in dieser Welt leben kann, ohne die ewige Seligkeit zu verlieren“ (Maurer, 1961, S. 9). Dieses „Interesse am Menschen“ (Heinzle, 1987, S. 87) stellt "eine Errungenschaft der neuen höfischen Kultur des 12. Jahrhunderts" (Heinzle, 1987, S. 87) dar.

\section{Sô wirbez, Rüedegêr, als liep als ich dir sî - Rüdiger als Werber Etzels}

In beiden Texten ist der Markgraf kein Hunne2, lebt jedoch mit seiner Frau Gotelind/ Gudelinda und seiner Tochter ${ }^{3}$ am Hof des hunnischen Königs Etzels. Rüdiger steht in einem vasallitischen Verhältnis: Er ist unter anderem mit der Burg Bechelaren/Bakalar belehnt. In seiner Stellung als Kronvasall unterstehen ihm ebenfalls Männer, beispielsweise der Grenzwächter Eckewart/Eckeward. Das Vorhandensein des Grenzwächters impliziert, dass der Markgraf über eine Grenzmark des hunnischen Reiches herrscht, mit deren Schutz er betraut zu sein scheint (Kunstmann, 1983, S. 235). Der in beiden Texten genannte Ort Bakalar/Bechelaren bezeichnet das heutige Pöchlarn (Paff, 1959, S. 30). Als Etzel erfährt, dass Kriemhild verwitwet wurde, beschließt er, um sie werben zu lassen. Rüdiger wird als Werber ausgewählt, er hân erkant von kinde di medelen künege (Schulze \& Grosse, 2011, V. 1144,4). Den angebotenen Lohn lehnt er ab (1150) (Panzer, 1955, S. 256-257) - denn Etzel fordert von Rüdiger keinen Zehnten, so dass dieser die Werbung als Leistung der fidelitas fasst. Das Zurückweisen des Lohnes ist eine "großmütige, vornehme Geste[,] charakterisiert ihn schon bei seinem ersten Auftreten

2 Obwohl der Markgraf kein Hunne ist, wird er durch Epitheta des Erzählers sprachlich diesen zugeordnet: Rüdiger und seine Männer werden di helde ûz Hiunen landen genannt (1163), gar Hiunen $(1180,1236)$. Denkbar ist, dass diese Exonyme die enge Bindung Rüdigers an seinen Lehnsherrn Etzel, sowie die erfolgreiche Inklusion des aus der Fremde stammenden Mannes in die hunnische Hofgesellschaft widerspiegeln, die deutlich in Formulierungen wie die Etzeln man (1248) und di recken hêr [...] ûz Etzeln lant (1255) gefasst sind. Diese Fremdheit der Familie und Rüdigers Gefolgsmannen wird mehrfach genannt der genutzte Begriff im Nl ist ellende $(1673,2141,2165)$-, auf seine Herkunft wird nicht eingegangen.

3 Im N/ wird kein Name der Tochter Rüdigers genannt, in der Klage heißt sie Dietlind. 
und führt ihn uns als den vollendet höfischen Mann vor" (Splett, 1968, S. 44). Im Markgrafen steht dem Rezipienten ein vorbildlicher Ritter gegenüber, der die im Tugendrittertum zentrale Tugend der mâze verkörpert. Wie bereits erläutert, stellt das Brautwerbungsschema eine Handlungsform der höfischen (Literar-)Gesellschaft dar, deren Verhaltenskodex und Literaturkanon sie eingeschrieben ist: Hier findet sich durch die Nutzung einer solchen Handlungsform die zweite Höfisierungssequenz. Die einzelnen Hauptstationen der Brautwerbung Etzels um Kriemhild zeigen eine dem Typus nach Schmid-Cadalbert entsprechende Schemaaktualisierung (Schmid-Cadalbert, 1985, S. 88). Anhand dieser kurzen Textsequenz entfaltet sich die Charakterisierung der Figur als tugendhaftem, die maze wahrendem Ritter in nuce.

\section{Nu sîn gote willekomen dies degene, der voget von Bechelâren unt alle sîne man - am Hof der Burgunden in Worms}

Vom "Machtbereich des Werbers" (Schmid-Cadalbert, 1985, S. 83) durch den die beiden Machtbereiche trennenden Raum - der im Falle des NI nicht wie meist als Meer aktualisiert ist, sondern als Festland -, kommt Rüdiger im „Machtbereich des Brautvaters bzw. -hüters" (Schmid-Cadalbert, 1985, S. 83) an: am Burgundenhof. „Von vornherein erwartet man von ihm nichts Nachteiliges" (Splett, 1968, S. 47), so wird Rüdiger herzlichst und nach dem Dürrenmatt'schen Schema empfangen: die Boten rüsten sich aus, um für ihren Herrn Ehre einzulegen (Schulze \& Grosse, 2011, V. 1170), sie kommen an (Schulze \& Grosse, 2011, V. 1172), werden von Verwandten des Königs empfangen (Schulze \& Grosse, 2011, V. 1174, 1181), Rüdiger tritt direkt vor den König und wird mit ehrenden Gesten empfangen (Schulze \& Grosse, 2011, V. 1182-1184), der König bittet um Überbringung der Botschaft und erteilt dem Boten die Erlaubnis, zu sprechen (Schulze \& Grosse, 2011, V. 1187), er bittet um die Erlaubnis, die Botschaft vorzutragen (Schulze \& Grosse, 2011, V. 1188), dies wird gewährt (Schulze \& Grosse, 2011, V. 1179), der Bote trägt die Botschaft nach bestimmten Grußbezeugungen stehend vor (Schulze \& Grosse, 2011, V. 1190-1192; Dürrenmatt, 1945, S. 26), man ist um die Verpflegung des Gefolges besorgt, solange sie auf Antwort zu warten haben (Schulze \& Grosse, 2011, V. 1173-1174, 1198). Einige Elemente des Empfangs heben Rüdiger jedoch aus der Stellung des Boten; dies nicht nur, da er ein höherstehender Bote als Wärbel und Swemmel ist (Splett, 1968, S. 47). Im Vergleich mit dem weiter oben nach Dürrenmatt (Dürrenmatt, 1945, S. 26) standardisiert dargestellten Botenempfangsschema und dem Empfang Wärbels und Swemmels fallen folgende Abweichungen auf: Rüdiger wird von des küniges næchsten mâge (Schulze \& Grosse, 2011, V. 1181) empfangen, statt von des küneges ingesinde (Schulze \& Grosse, 
2011, V. 1430); er kann ohne vorherige Nachricht direkt vor diesen treten (Schulze \& Grosse, 2011, V. 1182); König Gunther erhebt sich für den Freund (1182) und geleitet ihn an der Hand zuo dem sedele, dâ er selbe saz (Schulze \& Grosse, 2011, V. 1183-1184); Rüdiger verneigt sich nicht vor dem König, wie Wärbel und Swemmel dies tun (Schulze \& Grosse, 2011, V. 1437) - diese Abweichungen erstrecken sich über die Notwendigkeiten höfischen Empfangs und reflektieren die freundschaftliche Zugehörigkeit Rüdigers zum Burgundenhof. Sobald der König Rüdiger bittet, die Botschaft zu überbringen, ändert sich diese „Note ehrenvoller Herzlichkeit" (Splett, 1968, S. 47): Der Ablauf stimmt mit dem allgemeinen Dürrenmatt'schen Schema überein (Dürrenmatt, 1945, S. 25-26). ${ }^{4}$ Sobald der Fokus sich von der persönlichen Sphäre der Freundschaft zwischen den Burgundenkönigen und Rüdiger auf die seines Lehnsherrn Etzel verschiebt, werden „aus persönlichen Freunden wieder unpersönliche Boten“ (Dürrenmatt, 1945, S. 30). Es spiegelt die „äußre Form“ (Dürrenmatt, 1945, S. 19) des Verhaltens die „inner[...][e] Haltung" (Dürrenmatt, 1945, S. 19). Zum ersten Mal wird die doppelte Zugehörigkeit der Figur dargestellt: Eine rechtlich-bindende zu Etzel, und eine freundschaftliche, persönlichere, weniger stark bindende an die Burgunden. Kriemhild stimmt zunächst der Werbung nicht zu (Schulze \& Grosse, 2011, V. 1216,1), empfängt allerdings gerne den Rüdegêres lîp durch sîne manige tugende (Schulze \& Grosse, 2011, V. 1218, 2) - eine weitere, höfisierende Charakterisierung aus einem Figurenmund. Rüdiger bietet an, er wolde sie ergetzen, swaz ir ie geschach (Schulze \& Grosse, 2011, V. 1252). Nachdem Rüdiger den allbekannten und handlungskonstitutiven Eid geleistet hat (Schulze \& Grosse, 2011, V. 1255), stimmt Kriemhild der Werbung zu (Schulze \& Grosse, 2011, V. 1259-1261) - er verlässt hier seine Position als Brautwerber und verspricht statt Etzels minne âne leit, staete[r] vriuntschaft und friuntlîcher[r] liebe (Schulze \& Grosse, 2011, V. 1232, 1234) seinen Dienst (Toepfer, 2013, S. 213). An diesem Punkt im N/ ist eine "für den Fortgang des Epos und das Schicksal des Markgrafen wichtige Entscheidung [...] gefallen" (Splett, 1968, S. 52). Es stellt sich die Frage der Schuld: Hat der Markgraf sich mit diesem Eid an seinem späteren Konflikt schuldig gemacht? Diese Frage hat die Forschung oft gestellt und mannigfaltig beantwortet. So folgert Splett, Rüdiger sei durch seine Hilfsbereitschaft in das in der 37. Aventiure (Av.) dargestellte Dilemma geraten (Splett, 1968, S. 59). Wapnewski bewertet inn ebenso als unschuldig: Kriemhild habe einen Eid abgefordert,

4 Das Schema des Botenempfangs besteht aus den folgenden Elementen: „die Boten rüsten sich aus, um für ihren Herrn Ehre einzulegen, sie kommen an, werden vom Gesinde empfangen, das ihnen Herberge anweist, der Fürst erkundigt sich nach ihnen, sie treten vor die Hofgesellschaft, die sie mit bestimmten Gesten empfängt, sie bitten um Erlaubnis, ihre Botschaft ausrichten zu dürfen, man gewährt es ihnen, man ist um ihre Verpflegung besorgt, solange sie auf Antwort zu warten haben. Dieses Schema wird in jedem Einzelfall abgewandelt." (Dürrenmatt, 1945, S. 25-26). 
„deren Zweideutigkeit dieser so gerade denkende Mann nicht hatte durchschauen können" (Wapnewski, 1960, S. 389), die er daher auf zukünftiges Leid beziehen musste. Ähnlich denkt Weber: Rüdiger ahnte von der „Vieldeutigkeit des Eides [...] nicht das mindeste" (Weber, 1963, S. 98). Panzer hingegen meint, dass Rüdigers Pflichtenkonflikt „letzten Endes aus einer tragischen Schuld erwächst" (Panzer, 1955, S. 260), da er "die Widerstrebende beredete, die Werbung anzunehmen“ (Panzer, 1955, S. 260). Wiegmann sieht im Konflikt der 37. Av. den Topos vom „Untergang des Schuldlosen“ (Panzer, 1955, S. 189), entlastet Rüdiger von individuell-persönlicher Schuld und sieht die „sagenbedingte Grundlegung des Epos" (Panzer, 1955, S. 189) am Werk, die den Konflikt forme.

Anders verläuft die Werbung Attilas um Grimhild in der Ths. Rodingeir übernimmt in der Ths nicht die Funktion des Werbers Attilas, geschweige denn die der Figur, an der sich die Höfisierung entfaltet. Der Markgraf taucht erst auf, als die Niflungen auf ihrem Weg nach Susat des Markgrafen Burg Bakalar passieren.

Anhand der bisherigen Darstellung des Brautwerbungs- und des in ihm eingefassten Empfangsschemas kann das $\mathrm{NI}$ als helferzentrierte Brautwerbungsdichtung gefasst werden, ein Begriff, der Texte umfasst, die „die Darstellung der außergewöhnlichem Taten und Fähigkeiten des Helfers" (Schmid-Cadalbert, 1985, S. 204) in ihr Zentrum stellen dies tut das NI durch die sichtbare Aufwertung des vater[s] maneger tugende (Schulze \& Grosse, 2011, V. 2199).

\section{Di snellen Burgonden sich ûzhuoben - Die Reise der Burgunden nach Gran}

„An Rüdigers Residenz hat der Dichter [des NI] höfische Szenen angesiedelt, deren schwierige Konsequenzen sich erst später enthüllen“ (Schulze, 1997, S. 166). Relevant für diesen Artikel ist die Aufnahme der Burgunden in Bechelaren/Bakkalar. Der Markgraf wird in der 26. Av. (Schulze \& Grosse, 2011, V. 1635) wieder erwähnt. Hier begrüßt er die Burgundenkönige als "höfischer[r] Ritter par excellence" (Schulze, 1997, S. 162) mit einer "gastlichen Aufnahme" (Schulze, 1997, S. 162) auf der Burg Bechelaren. Nach der beschwerlich-kargen und gefährlichen Reise treffen die Burgunden auf den Grenzwächter Eckewart, der den Markgrafen und seine Gastfreundschaft als frappierende Gegensätze zum letzten Stück der Reise zeichnet: An den minnesang'schen Topos des locus amoenus anklingend, schildert Eckewart seinen Herrn als tugendhaft, alsô der süeze meie daz gras mit bluomen tuot (Schulze \& Grosse, 2011, V. 1636). Er bezeichnet ihn als freigiebig: swenne 
er sol helden dienen, sô ist er vrœlich gemuot (Schulze \& Grosse, 2011, V. 1636). Wird der locus amoenus typischerweise als vom höfischen Leben abgegrenzter Gegenort konzipiert, beispielsweise in den Epen Gottfrieds von Straßburg Tristan, sowie Hartmanns von Aue Erec, ist im Falle Bechelarens interessant und hervorzuheben, dass Eckewart die Residenz des Markgrafen Rüdigers in der Terminologie dieses Topos beschreibt: er spricht vom Frühlingswonnemonat Mai, der das Gras mit Blumen ziert (Schulze \& Grosse, 2011, V. 1636); dass also Höfisches - Rüdiger ist ein idealtypischer, vorbildlicher Ritter, der höfisch residiert und lebt -, und Natürliches, von der Sphäre des Höfischen sonst Abgetrenntes, zueinander geführt werden. Der Erzähler schafft hier eine Höfisierung auf zwei Ebenen: Einerseits durch die Implikation eines literarischen Schemas - des locus amoenus -, andererseits durch die weitere Definierung Rüdigers als Tugendritter und die Differenzierung entsprechender Eigenschaften aus einem weiteren Figurenmund. Der Empfang entfaltet sich gemäß höfischem Zeremoniell, wobei Rüdiger die Burgunden mit schemaübersteigender Gastfreundschaft empfängt: Er sorgt nicht nur schemakonform für Unterkunft und Verpflegung der Gäste, sondern verspricht die sicherere Aufbewahrung ihrer Pferde und Ausrüstung (Schulze \& Grosse, 2011, V. 1656) und, sollte doch etwas verloren gehen, Reparation für alles, swaz ir hie verlieset (Schulze \& Grosse, 2011, V. 1657); insgesamt gilt: den unkunden gesten diente man hêrliche sît (Schulze \& Grosse, 2011, V. 1668). Mithilfe der Höfisierungsstrategie des Empfangsschemas „schildert der Dichter das höfische Leben [en detail] und läßt langsam eine immer gelöstere Atmosphäre entstehen“ (Splett, 1968, S. 63), bis Hagen vorschlägt, der junge Giselher solle die Tochter des Markgrafen heiraten. Dieser stimmt diesem Vorschlag zu, auch die Tochter willigt in die Verlobung ein. Hier stärkt Rüdiger seine doppelte Zugehörigkeit zu seinem späteren Nachteil weiter: Er verschwägert sich mit Giselher und stellt eine verwandtschaftliche Bindung her. Durch die Bitte des Markgrafen und um seine Gastfreundschaft zu ehren (Splett, 1968, 64), bleiben die Könige unz an den vierden morgen (Schulze \& Grosse, 2011, V. 1688). Vor der Abreise beschenkt der Gastgeber die Burgunden ausführlich und großzügig - diese nehmen die Geschenke an und erweisen Rüdiger somit Ehre. Erneut erwähnt der Erzähler die Geberfreudigkeit und charakterisiert den Markgrafen wiederholt „als hochsinnigen Ehrenmann“ (Weber, 1963, S. 85) (Schulze \& Grosse, 2011, V. 1689). Nun machen sich die Burgunden unter dem Geleit Rüdigers auf den Weg nach Gran: Ich wil iu selbe leiten und heizen wol bewarn, daz iu ûf der strâze niemen muge geschaden (Schulze \& Grosse, 2011, V. 1705).

Auch in der Ths werden die drei Könige und Högni an Attilas Hof eingeladen und folgen dieser Einladung, ebenso bietet Rodingeir ihnen im Zuge seines ersten Auftauchens 
im Text in Bakalar ein Unterkommen. Allerdings ist diese Textsequenz abweichend von der im NI gestaltet: „Die Saga berichtet davon kurz und knapp in zwei Sätzen, während das N[ibelungenlied] freudig die sich hier bietende Gelegenheit ergreift, [...] allen höfischen Prunk zu entfalten“ (Wiesniewski, 1961, S. 98). Hagen stößt auch in der Ths auf den schlafenden Grenzwächter Eckeward. An dieser Stelle finden sich die ersten Charakterisierungen Rodingeirs im Text der Ths. Eckeward nennt seinen Herrn einen guten Gastgeber und beschreibt: „Er ist ein guter Fürst“ (Erichsen, 1967, S. 392). Wie Im NI reitet Rodingeir den Ankömmlingen entgegen und empfängt "die Niflungen freundlich und lud sie ein, seine Gäste zu sein“ (Erichsen, 1967, S. 392). Abweichend stellt sich die Verlobung dar: Rodingeir beschließt, seine Tochter an Giselher zu geben; einer Zustimmung der Braut bedarf es nicht. In diesem Verhalten wird eine weitere Ebene der Höfisierung im $\mathrm{NI}$ offenbar: „Eine derart unhöfische Auffassung von der Frau, die wie eine Sache behandelt und mit den Gastgeschenken in eine Reihe gestellt wird, findet sich nicht im mittelhochdeutschen Epos (Splett, 1968, S. 67). Die implizite Werbung um die Tochter des Markgrafen und die Notwendigkeit ihrer Zustimmung können als höfisierende Elemente gewertet werden. Und so „erwächst aus der Gastfreundschaft im höfischen Rahmen eine neue verwandtschaftliche Beziehung, die das alte freundschaftliche Verhältnis zwischen Rüdiger und den Burgunden auf eine neue Ebene überführt" (Toepfer, 2013, S. 224). Am nächsten Morgen reisen die Niflungen beschenkt weiter. Rodingeir gewährt den Burgunden Geleitschutz. In der Ths wird im Gegensatz zum NI "auf eine höfische Überhöhung [...] kein Wert gelegt" (Toepfer, 2013, S. 224): Der Erzähler der Ths fokalisiert extern: „Der Sagaschreiber berichtet nur das, was ein Außenstehender sieht und hört" (Splett, 1968, S. 55). Im NI hingegen findet sich ein nullfokalisierender Erzähler. Der Erzähler des NI setzt die so möglich gewordene Darstellung psychologischer Figurenzustände als Höfisierungsstrategie ein: An späterer Stelle wird so die Darstellung des Konflikts aus einer dem höfischen „Interesse am Menschen“ (Heinzle, 1987, S. 87) und schließlich der im Rahmen der "Verchristlichung des literarischen Lebens" (Heinzle, 1987, S. 87) der höfischen Gesellschaft aufgeworfenen Frage der Vereinbarkeit von christlich-jenseitigen und weltlich-diesseitigen Ordnungsvorstellung möglich, während dies in der Ths durch die externe Fokalisierung nicht möglich ist.

\section{8. sô hân ich bôsliche unde vil ubele getân - Rüdigers Konflikt}

„Die 37. Av[.] ist Rüdigers Dilemma gewidmet." (Schulze, 1997, S. 168) Auf eine Absprache der höfischen Tugend der Kühnheit (Schulze \& Grosse, 2011, V. 2137), sowie der fidelitas Etzel gegenüber reagiert die Figur mit bisher nicht gezeigter Gewalt: Er sluoc 
sô kreftliche den hiunischen man, daz er im vor den fuozen lac vil schier tôt (Schulze \& Grosse, 2011, V. 2139). Diese Textstelle illustriert als Vasallitätskonflikt (Müller, 1998, S. 310) gefasst die Intensität, mit der „die Ehre als treibende Kraft im Nibelungenlied wirksam ist" (Müller, 1998, S. 310): Ehre und höveschkeit konstituieren wie die Lehnbindung (Wiegmann, 1982, S. 189) wirklichkeitsbestimmende Seinsrealitäten. Wird höveschkeit aberkannt, geraten Ritter in einen Bereich „außerhalb höfischer mâze" (Schulze, 1997, S. 169), "[w]o es keine höfische Repräsentation gibt, gibt es auch keinen funktionierenden Hof" (Schulz, 2015, S. 66). Ex negativo findet sich eine Höfisierung: Durch die fehlende höveschkeit wird eine gewalttätig-unberechenbare Situation erzeugt und damit die Notwendigkeit der durch den Erzähler eingesetzten Höfisierungsstrategien zur Aufrechterhaltung der gesellschaftlichen Ordnung unterstrichen. Auch, wenn an späterer Stelle diese Ordnung in Kontrast mit der christlich-jenseitigen gesetzt wird, demonstriert der Erzähler an dieser Stelle die Notwendigkeit der weltlichen Ordnung und die Gefahr ihres Fehlens. Rüdiger erkennt nun sein Dilemma; die bereits im Rahmen des Empfangs besprochene Doppelzugehörigkeit der Figur zur Sphäre der Burgunden und der des Hunnenkönigs geht nun also über die Verschärfung derselben über (rechtliche) Bindungen und sich ausschließende Tugendideale im Pflichtenkonflikt auf: $\mathrm{Er}$ "muss sich im Nibelungenlied zwischen seiner Lehnspflicht und der Treue gegenüber seinen vriunden entscheiden“ (Toepfer, 2013, S. 211), wobei ihn beide Bindungsarten als einen tugendhaften und edlen Ritter kennzeichnen (Toepfer, 2013, S. 219). Im Rahmen des Pflichtenkonflikts sind rechtliche Bindungen von „seelisch-sittliche[n] Bindungen“ (Wapnewski, 1960, S. 393), die auf menschlich-emotionalem Grund wie Familienbeziehungen beruhen, und weniger starke rechtliche Implikation tragen, zu scheiden ${ }^{5}$. Hatte sich weiter oben gezeigt, dass die höfisch-ritterlichen Tugenden auf christlichen Geboten fußen und eine hochmittelalterliche Überformung und im Zuge dessen eine weitere Bedeutungsebene erfahren haben, so befindet sich der Markgraf nun im Konflikt mit beiden Ebenen der Tugend triuwe: Er kann entweder der weltlich-rechtlichen Implikation in Form der Erfordernisse der vasallitischen Bindung nachkommen, oder der weniger stark rechtlichen, emotional-sittlichen, die aus den im Verlauf des Textes geschlossenen Bindungen an die Burgunden folgt. Für welche triuwe er sich entscheidet, er wiste schaden gewinnen und ungefüegiu leit (Schulze \& Grosse, 2011, V. 2153): Der Markgraf entschließt sich nach Reflexion - nullfokalisierend-psychologisierend dargestellt - , , der Lehnspflicht

5 So ist beispielsweise der rechtlich-bindende Teil des Geleitschutzes auf die Reisezeit beschränkt und somit sind "denn die rechtlichen Wirkungen der Geleitleistungen [...] längst beendet" (Wapnewski, 1960, S. 393). Dies auch, wenn Rüdiger diese Bindung als rechtlich-bindendes Kampfeshindernis angibt: "Für ihn ist diese Rechtshandlung dem Gast gegenüber eine feste Bindung, ohne die er sich längst am Kampf beteiligt hätte." (Schulze \& Grosse, 2011, S. 858). 
zu folgen [Schulze \& Grosse, 2011, V. 2160-2163]; das ist unter rechtlichen Gesichtspunkten die einzige Lösung des Konflikts" (Schulze \& Grosse, 2011, S. 860): Ich muoz iu leisten, als ich gelobt hân (Schulze \& Grosse, 2011, V. 2163) fasst Rüdiger zusammen. Mit der bereits explizierten, durch Bumke nachgezeichneten Ausdifferenzierung der Tugendbedeutungen, allen voran der Bedeutung der triuwe, und mit Wapnewski nimmt diese Arbeit eine Hierarchisierung der Verpflichtungen (Wapnewski, 1960, S. 54) an, wobei die rechtliche Bindung an Etzel priorisiert wird und werden muss. Der genannten Hierarchisierung der Bindungen folgt eine Entschuldung Rüdigers, denn er muss sich nicht „zwischen zwei gleichrangigen Werten entscheiden“ (Toepfer, 2013, S. 231-232), sondern folgt der "Konsequenz eines juristisch eindeutigen Sachverhalts" (S. 231). Auch die im Folgenden beleuchtete Textstelle und das Verhalten der Figuren weist in diese Richtung.

Der Markgraf und seine Männer werden gewaffnet und ziehen zum Schauplatz der Kämpfe (Schulze \& Grosse, 2011, V. 2166-2168): „Rüdiger [wird] [...] als Lehnsmann Etzels [...] kämpfen" (Schulze \& Grosse, 2011, S. 861). Auch, wenn eine Hierarchisierung der Bindungstypen angenommen wird, so ist der Markgraf aufgrund seiner doppelten Zugehörigkeit zu den Sphären beider Typen nicht aus der Tragik des Konflikts entlassen. Die Darstellung der psychologischen Zustände während der Gespräche mit den Burgunden macht deutlich, dass er die freundschaftlich-sittliche triuwe nicht aufkündigen möchte, es jedoch aus rechtlich-bindender Sicht muss. Kurz vor Kampfbeginn bittet Hagen um des Markgrafen Schild: Die Schildgabeszene entfaltet sich. Dass diese Bitte "nicht auf der realistischen Handlungs- und Erzählebene liegt" (Hoffmann, 1987, S. 72), ist leicht erkennbar, könnte sich Hagen doch bei den Schildern der Gefallenen bedienen. „Hagen hat erkannt, daß Rüdiger wider Willen im Begriff ist, die triuwe gegen seine Verwandten und Freunde zu verletzen" (Harms, 1963, S. 42) - Hagen ermöglicht also, wenn auch limitiert, beide triuwen zu halten. Die Schildgabe durch Hagen weist auf die Hierarchisierung beider Bindungsarten respektive triuwe-Ebenen und einer folgenden Entschuldung Rüdigers, da dieser der priorisierten triuwe-Bindung folgen muss: Würde der Markgraf einer gleichwertigen Bindung folgen, so müsste er die zweite aufgeben, die Bindung an die Burgunden könnte nicht weiterbestehen. Im NI nun bestehen jedoch beide Bindungen weiter, eine parallele Existenz beider ist präkonfliktiv möglich, und wird auch unter dem akuten Konflikt durch Hagens Schildbitte nicht aufgelöst: In weltlicher Ordnung und der Etzel zugehörigen Figurensphäre scheint der Konflikt mit der Priorisierung der rechtlichen Bindung gelöst, in freundschaftlich-sittlicher, den Burgunden zugehöriger Sphäre, die auf einer - wie bereits genannt wurde -, christlichen 
Grundlage fußt, ist dem auch nach der versöhnlichen Schildgabe nicht so, besteht der Konflikt gar weiter als tragischer fort: „In der Konsequenz [der Schildgabe] bleibt der tragische Konflikt, wenn er aufgrund seiner Unlösbarkeit als solcher bezeichnet wird, unverändert bestehen" (Toepfer, 2013, S. 233). Wie bereits seit seinem Empfang am Burgundenhof vereint Rüdiger zwei Zugehörigkeiten in sich - war die Auslebung beider bisher zeitlich getrennt, so geschieht sie nun gleichzeitig und trägt so zur Unlösbarkeit des Konflikts auch im Angesicht scheinbarer Lösungen bei. Hagen drückt durch seine Bitte sicherlich Zustimmung zur Entscheidung des Markgrafen, Etzel die fidelitas zu halten, aus; er zeigt sich gleichzeitig affirmativ einer Hierarchisierung der Bindungen und ihrer unterschiedlich sichtbaren Realisierung gegenüber und trägt dazu bei, diese doppelte Bindung, gar die Doppelzugehörigkeit Rüdigers aufrecht zu erhalten. Wie die Bindungen Rüdiger innerhalb der christlich-jenseitigen Ordnung betreffen, erkundet der nächste Punkt dieses Artikels.

Nun beginnen die Kämpfe: den schilt huob Rüedegêr. des muotes er ertobete [...] manegen slac vil swinden sluoc der margrâve rîch (Schulze \& Grosse, 2011, V. 2203), es entbrennt der Zweikampf zwischen Gernot und Rüdiger (Schulze \& Grosse, 2011, V. 22132215). Mit sechs Strophen, also 24 Versen (Schulze \& Grosse, 2011, V. 2213-2218) ist er knapp dargestellt, vor allem im Gegensatz zu den ihn prädizierenden Dialogen. Diese nehmen 32 Strophen, oder, anders gesagt, 128 Verse (Schulze \& Grosse, 2011, V. 21712202) ein. Harms nennt die Szene aufgrund ihrer Kürze gar "unhöfisch" (Harms, 1963, S. 43). Diese Einschätzung passt zu der Beobachtung, dass hier nicht der vater maneger tugende (Schulze \& Grosse, 2011, V. 2199), Freund der Burgunden, kämpft, sondern ein seiner Pflicht nachkommender Ritter.

\section{Ouwê got von himele - Höfisierung durch christliche Elemente}

Weiterhin wird die bereits beleuchtete Gespaltenheit der Figur in einen christlichen Kontext gebettet. Vor der Folie der bereits dargelegten "Verchristlichung der Literatur" (Maurer, 1961, S. 6) kann die 37. Av. des NI gelesen werden. Der Markgraf trennt mit êre unde lîp auf der einen, und sêle auf der anderen Seite weltliche von christlich-jenseitigen Werten - er habe Kriemhild geschworen, dass er wâgete êre unde ouch den lîp (Schulze \& Grosse, 2011, V. 2147), nicht jedoch, für sie sein Seelenheil zu wagen: daz ich di sêle vliese, des enhân ich niht gesworn (Schulze \& Grosse, 2011, V. 2147). Er findet sich allerdings in einer Situation, in der dies verlangt wird: Das Treulosseinmüssen ist im Angesicht beider Treue fordernden Bindungen „nicht mehr nur Schande vor den Menschen, [...] sondern 
es ist auch Sünde vor Gott. Nicht nur die Ehre geht dem Treulosen verloren, sondern auch das Seelenheil“ (Maurer, 1961, S. 34). Mit der Priorisierung der innerweltlichen Bindung ergibt sich, dass für Rüdiger „ein Leben in [...] [christlicher] Ordnung nicht mehr möglich ist" (Maurer, 1961, S. 36), hat er doch die christlich konnotierte Ebene der triuwe verletzt. Dies auch, wenn Hagen ihm nach der Schildgabe versichert: Nu lôn iu got der gâbe (2198), und Etzel ihm Gottes Lohn für die Einhaltung der fidelitas wünscht (Schulze \& Grosse, 2011, V. 2162), bleiben doch bedeutungsschwer mehrere Gottesanrufungen des Markgrafen unerhört (Schulze \& Grosse, 2011, V. 2150-2151, 2189) - es geschieht keine den Konflikt lösende, göttliche Intervention. Während die Hauptfiguren des NI von dieser Korrelation ihres Handelns mit christlicher Perspektive ausgespart bleiben, dient es im Falle des Markgrafen dazu, "die [seit dem Erec gestellte] Frage, ob das Handeln der Protagonisten vor Gott bestehen könne, ob sie Gottes Gnade erlangen“ (Schulze, 1997, S. 171) zu behandeln. Die höfische Literatur war also nicht nur am Rande mit Fragen der Vereinbarkeit der Sphären des christlich-jenseitigen und des weltlich-höfischen beschäftigt; mit der Behandlung der Frage, wie gelebt werden solle, erhält die außerliterarische Welt einen Reflex in der Dichtung. Die Figur des Markgrafen ist ein Beispiel für folgenden Umstand: „Das Schicksal der Einzelseele, das Schicksal des Nächsten vor Gott, ohne Rücksicht auf Hof und Welt, nur davon kann [in der Literatur des 13. Jahrhunderts] glaubhaft gehandelt werden." (Bertau, 1983, S. 107). Die "Gestaltung der inneren Krise als Gewissenskonflikt mit christlicher [und weltlicher] Perspektive ist ein weiteres Beispiel der mehrfach ausgewiesenen höfisierenden Tendenzen des Nibelungenliedes" (Schulze, 1997, S. 171).

Die Ths "kennt die Schildbitte und -übergabe nicht [...] Sie weiß auch - trotz Freundschaft und Verschwägerung - nichts von einer Pflichtenkollision im Markgrafen Rodingeir" (Wapnewski, 1960, S. 402). Auch baut die Ths keine christlichen Elemente ein. Hier findet der Eingriff Rodingeirs in die bereits entbrannten Kämpfe der Hunnen mit den Niflungen als Folge Herzog Blodlins Tod durch Gernoz statt (Wisniewski, 1961, S. 165) - er will aus Rache die Niflungen erschlagen (Erichsen, 1967, S. 408). Später wird Rüdiger von Giselher mit dem Schwert erschlagen, das dieser ihm in Bakalar geschenkt hatte (Erichsen, 1967, S. 410) - sein Tod ist Rache für die durch seinen Eingriff in den Kampf mit den Niflungen gebrochene Verschwägerung. Dabei erwähnt „die Saga mit keinem Wort die menschliche Tragik [...], die in der Paarung Rodingeir - Giselher liegt" (Wiesniewski, 1961, S. 166), ebenso findet keine Darstellung psychologischer Zustände statt: „Kein Wort davon, daß irgendein Niflung oder Hunne über das eben geschehene Unglück erschüttert sei“ (Wiesniewski, 1961, S. 167). Die Darstellung psychologischer Zustände, sowie die 
Reflexion über die triuwe-Bindungen sind nicht notwendig, da der Konflikt nicht vorkommt, gar durch das bereits explizierte Erzählerverhalten nicht zustande kommen, oder höchstens durch die Außensicht ausgedrückt werden könnte. Es lässt sich erkennen, dass die Schilderung des Niflungenuntergangs in der Ths - wie bereits während der Betrachtung der vorherigen relevanten Textstellen offenbar wurde -, einem vollkommen anderen Erzählerverhalten unterworfen ist als die entsprechende Textsequenz im NI, woraus sich die unterschiedliche Darstellung psychologischer Figurenzustände, dem vorgängig die Möglichkeit dazu, ergibt.

\section{Ergebnisse}

Dieser Artikel hat sich mit der Figur des Markgrafen Rüdiger von Bechelaren im NI und in der Ths befasst. Nachdem er nach den textexternen Faktoren des Überlieferungsstands und eines Figurenvorbilds gefragt hat, hat er die Charakteristika der Figur in beiden Texten kontrastierend erarbeitet. Hierbei fiel eine Reihe an Unterschieden auf. Obwohl der Markgraf Rüdiger nicht zu den Hauptfiguren der Texte gehört, spielt er im NI eine zentrale Rolle, werden ihm das gesamte Werk betreffende, handlungsrelevante Passagen und textgestalterische Elemente zugeschrieben, werden an ihm zentrale (außer) literarische Konflikte verhandelt (Schulze, 1997, S. 163). Er fungiert als Werber, der Etzels Brautwerbung um Kriemhild ausführt; er ist freigiebiger Gastgeber der Burgunden auf ihrem Weg nach Gran; er wird porträtiert als eine in einen Konflikt geratene Figur, die diese Situation reflektiert und einen Ausweg sucht (Schulze, 1997, S. 162). Auf einer höheren Ebene als der der individuellen Figur und seiner Charakteristika fungiert der Markgraf als Schauplatz der Höfisierung, an dem die einzelnen Höfisierungsstrategien sich entfalten und so die Höfisierung des Gesamtwerkes herstellen. Dies ermöglicht erstens ein im Gegensatz zur Ths nullfokalisierendes Erzählerverhalten, das eine Darstellung psychologischer Zustände der Figur, und letztlich die Inkorporation der die höfische Gesellschaft im Hochmittelalter beschäftigenden Frage der Vereinbarkeit von christlicher und weltlicher Lebensführung erlaubt; zweitens die Nutzung der höfischliterarischen Elemente des locus amoenus, der,äußern Formen' des Brautwerbungs- und Botenempfangsschemas, sowie Teilen des Konzeptes des Tugendrittertums (Dürrenmatt, 1945, S. 25-26). Die literarische Behandlung der Frage "was ist der Sinn unseres Daseins in der Welt und wie geben wir ihm seinen Sinn?" (Maurer, 1961, S. 8), die weltlich-höfischritterliche Werte mit den jenseitig-christlichen korreliert, wird im NI für ein höfischchristliches Publikum behandelt, während dies in der Ths - möglicherweise, da das Publikum ein anderes ist -, nicht geschieht. 
Begutachtung: Extern begutachtet.

Interessenkonflikt: Es besteht kein Interessenkonflikt.

Finanzielle Förderung: Dieser Beitrag wurde von keiner Institution finanziell unterstützt.

Peer-review: Externally peer-reviewed.

Conflict of Interest: The author has no conflict of interest to declare.

Grant Support: The author declared that this study has received no financial support.

\section{Literaturverzeichnis}

Bertau, K. (1983). Über Literaturgeschichte. Literarischer Kunstcharakter und Geschichte in der höfischen Epik um 1200. München: Beck.

Breulmann, J. (2009). Erzäh/struktur und Hofkultur. Weibliches Agieren in den europäischen IweinstoffBearbeitungen des 12. bis 14. Jahrhunderts. Münster: Waxmann.

Bumke, J. (2005). Höfische Kultur. Literatur und Gesellschaft im hohen Mittelalter. München: Deutscher Taschenbuch Verlag.

Erichsen, A. (1967). Die Geschichte Thidreks von Bern. Darmstadt: Wissenschaftliche Buchgesellschaft.

Dürrenmatt, N. (1945). Das Nibelungenlied im Kreis der höfischen Dichtung. Bern: Lang.

Ehrismann, O. (2002). Nibelungenlied. Epoche - Werk - Wirkung. München: Beck.

Genette, G. (1994). Die Erzählung. München: Fink.

Haferland, H. (2013). Psychologie und Psychologisierung. Thesen zur Konstitution und Rezeption von Figuren. Mit einem Blick auf ihre historische Differenz. In F. Kragl, \& C. Schneider (Hrsg.), Erzähllogiken in der Literatur des Mittelalters und der Frühen Neuzeit. Akten der Heidelberger Tagung vom 17. bis 19. Februar 2011 (S. 91-118). Heidelberg: Universitätsverlag Winter.

Harms, W. (1963). Der Kampf mit dem Freund oder Verwandten in der deutschen Literatur bis um 1300. München: Eidos.

Heinzle, J. (1987). Das Nibelungenlied. Eine Einführung. München: Artemis.

Heinzle, J. (2014). Traditionelles Erzählen. Beiträge zum Verständnis von Nibelungensage und Nibelungenlied. Stuttgart: Hirzel.

Hoffmann, W. (1987). Das Nibelungenlied. Grundlagen und Gedanken zum Verständnis erzählender Literatur. Frankfurt am Main: Diesterweg.

Jacobsen, P. C. (1965). Die Quirinalien des Metellus von Tegernsee. Leiden: Brill.

Kramarz-Bein, S. (2002). Die Piðreks saga im Kontext der altnorwegischen Literatur. Tübingen: Francke.

Kunstmann, H. (1983). Wer war Rüdiger von Bechelaren? Zeitschrift für deutsches Altertum und deutsche Literatur, $112,(4), 233-252$.

Lachmann, K., Haupt, M., Vogt, F. \& Kraus, C. (1970). Des Minnesangs Frühling. Stuttgart: Hirzel. Lienert, E. (2015). Mittelhochdeutsche Heldenepik. Eine Einführung. Berlin: Schmidt. 
Maurer, F. (1961). Leid. Studien zur Bedeutungs- und Problemgeschichte besonders in den grossen Epen der staufischen Zeit. Bern: Francke.

Morgan, Q. B. (1912). Rüedegér. Beiträge zur Geschichte der deutschen Sprache und Literatur. 37 (2), 325-336.

Müller, J. (1998). Spielregeln für den Untergang. Die Welt des Nibelungenliedes. Tübingen: Niemeyer.

Müller, J. (2015). Das Nibelungenlied. Berlin: Schmidt.

Paff, W. J. (1959). The Geographical and Ethnic Names in the Piðriks Saga. A Study in Germanic Heroic Legend.

's-Gravenhage: Mouton and Co.

Panzer, F. (1955). Das Nibelungenlied. Entstehung und Gestalt. Stuttgart: Kohlhammer.

Pidal, R. M. (1937). Das Spanien des Cid. München: Hueber.

Reichert, H. (1992). Heldensage und Rekonstruktion: Untersuchungen zur Thidrekssaga. Wien: Fassbaender.

Ritter-Schaumburg, H. (1981). Die Nibelungen zogen nordwärts. München: Herbig.

Röhrich, L. (1988). Orale Traditionen als historische Quelle. Orale Traditionen als historische Quelle. Einige Gedanken zur deutschsprachigen mündlichen Volkserzählung. In J. Ungern-Sternberg, \& H. Reinau (Hrsg.), Vergangenheit in mündlicher Überlieferung (S. 79-99). Stuttgart: Teubner.

Schmid-Cadalbert, C. (1985). Der Ortnit AW als Brautwerbungsdichtung. Ein Beitrag zum Verständnis mittelhochdeutscher Schemaliteratur. Bern: Francke.

Schulz, A. (2015). Erzähltheorie in mediävistischer Perspektive. Studienausgabe. Berlin: de Gruyter.

Schulze, U. (1997). Das Nibelungenlied. Stuttgart: Reclam.

Schulze, U., \& Grosse, S. (2011). Das Nibelungenlied. Mittelhochdeutsch/Neuhochdeutsch. Stuttgart: Reclam.

Splett, J. (1968). Ruediger von Bechelaren. Studien zum zweiten Teil des Nibelungenliedes. Heidelberg: Universitätsverlag Winter.

Toepfer, R. (2013). Höfische Tragik. Motivierungsformen des Unglücks in mittelalterlichen Erzählungen. Berlin: de Gruyter.

Wapnewski, P. (1960). Deutsche Literatur des Mittelalters. Ein Abriß. Göttingen: Vandenhoeck und Ruprecht. Wapnewski, P. (1960). Rüdigers Schild. Zur 37. Aventiure des Nibelungenliedes. Euphorion. Zeitschrift für Literaturgeschichte, 4(54), 380-410.

Weber, G. (1963). Das Nibelungenlied. Problem und Idee. Stuttgart: Metzler.

Wiegmann, H. (1982). Rüdiger von Bechelaren, Max Piccolomini und Winnetou. Beobachtungen zum Topos vom Untergang des Schuldlosen. Jahrbuch der Karl-May-Gesellschaft. 12, 185-195.

Wiesniewski, R. (1961). Die Darstellung des Niflungenunterganges in der Thidrekssaga. Tübingen: Niemeyer. 
